\title{
The depression of operant behavior and retarding action on discrimination learning by amphetamine
}

\author{
SHERWOOD O. COLE \\ RUTGERS UNIVERSITY ${ }^{I}$
}

Amphetamine, $2 \mathrm{mg} / \mathrm{kg}$ administered on four trials one week apart, depressed intermittently reinforced operant behavior and retarded food-motivated discrimination. Since a recovery of operant rate occurred on a subsequent non-drug trial with no similar improvement in discrimination, the drug appeared to retard the learning of discrimination rather than temporarily repressing it as a mere by-product of the effect on overall operant rate.

The depressant action of amphetamine on "foodmotivated" performance has been reviewed elsewhere (Cole, 1967). There is evidence that this action breaks down under conditions where greater discrimination is required to procure reinforcement. For example, Kelleher et al (1961) found an increase rather than a decrease in performance with amphetamine under conditions of differential reinforcement of low rates (DRL), and Ferster et al (1962) found a similar increase in performance under FR schedules with pre-time-out.

The present study further investigates the action of the drug under conditions where the $S$ must exercise discrimination to procure reinforcement. Special attention is focused on: (1) the general effect of amphetamine on intermittently reinforced operant behavior, and (2) the specific effect of amphetamine on the learning of a food-motivated discrimination. Subjects

Sixteen male albino rats, purchased from West Jersey Biological Supply and approximately 100 days old at the beginning of training, served as Ss. They were housed in a temperature-controlled laboratory with water and Purina chow available ad lib in the home cage.

\section{Procedure}

Initially, all $\mathrm{Ss}$ were given 20 min training sessions in a standard Skinner box (Gerbrands) under $30 \mathrm{~h}$ food deprivation. Training was continued until each $\mathrm{S}$ had established a baseline rate, with approximately one week allowed between sessions. The 16 Ss were randomly assigned to either a drug or a control group. Statistical analysis indicated no significant difference in the baseline rates of the groups as a result of assignment.

Each $\mathrm{S}$ was given one $1 / 2 \mathrm{~h}$ test trial a week for five consecutive weeks. Reinforcement conditions were manipulated by dividing the $1 / 2 \mathrm{~h}$ trial into six periods of $5 \mathrm{~min}$ each. On Trials 1 and 2, three of the six periods were randomly chosen for reinforcement (R) with the restriction that no more than two consecutive periods be so defined. During the $5 \mathrm{~min}$ $\mathbf{R}$ periods, Ss were continuously rewarded with a single $45 \mathrm{mg}$ food pellet ${ }^{2}$ for each bar press, whereas the remaining periods were nonreinforced (NR). On Trials 3, 4, and $5, R$ and NR were alternated on the six successive periods with half of the $S s$ in each group assigned the $R, N R, R, N R, R, N R$ sequence and the remaining the $N R, R, N R, R, N R, R$ sequence. On all trials, a cue light, directly above the food tray, remained on the entire $R$ period to facilitate discrimination between $R$ and NR.

The test procedure on Trials 1-4 was as follows. After being food deprived for $24 \mathrm{~h}$, the $\mathrm{S}$ was removed from the home cage, weighed, and injected intramuscularly. Drug Ss received $2 \mathrm{mg} / \mathrm{kg}$ of amphetamine $^{3}$ with the controls receiving distilled water similarly adjusted to body weight. Fifteen min later, the $S$ was put in the apparatus, and the number of bar presses for each successive $5 \mathrm{~min}$ period was measured and a cumulative record made of the total presses during the trial. Trial 5 served as a "recovery-from-drug" trial with the drug Ss recelving a distilled water injection instead of amphetamine. In every other respect, the procedure was identical to the previous trial. At the conclusion of a trial, the $S$ was immediately returned to the home cage. Ss were tested on the same day of the week on successive trials to assure a uniform period between trials.

\section{Results and Discussion}

The total number of bar presses for the two groups on successive trials is presented in Fig. 1. A trend analysis of Trials $1-4$ yielded a significant Drug effect ( $F=12.87$, df $=1 / 14, p<.005)$, a significant difference between Trials $(F=27.77, d f=3 / 42, p<.001)$, and a significant Drug by Trials interaction ( $F=9.37$, $\mathrm{df}=3 / 42, p<.001$ ). An analysis of Trial 5 recovery data indicated no significant difference between groups.

The percent of bar presses made during the $R$ periods by the two groups on successive trials (measure of discrimination) is presented in Fig. 2. A trend analysis of Trials 1-4 indicated a significant Drug effect only $(F=11.75$, df $=1 / 14, p<.005)$. Analysis of Trial 5 recovery data indicated that a significant difference in the percent of responses during $R$ periods by the two groups was still present when the drug Ss no longer received amphetamine $(F=$ 22.56, $\mathrm{df}=1 / 14, \mathrm{p}<.001$ ).

The present study demonstrates the effectiveness 


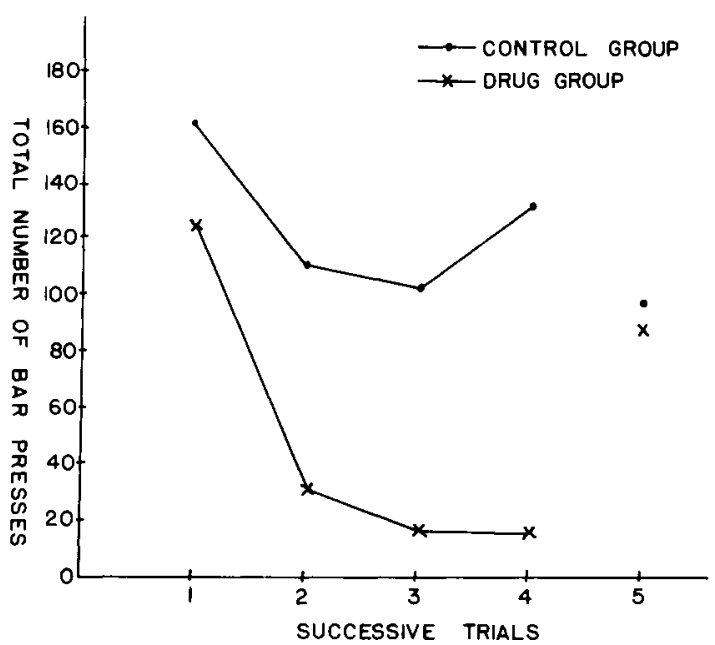

Fig. 1. Mean number of total responses ( $R$ and NR periods) by drug and control groups on successive trials.

of amphetamine in depressing general operant behavior intermittently reinforced by food. Since the procedures of Kelleher et al (1961) and Ferster et al (1962) differed from that employed in the present study, their findings are not necessarily in conflict with those reported here. However, they do point out the need for a careful investigation of the specific discriminatory conditions under which the depressant effect of the drug is present and those where there is an apparent breakdown.

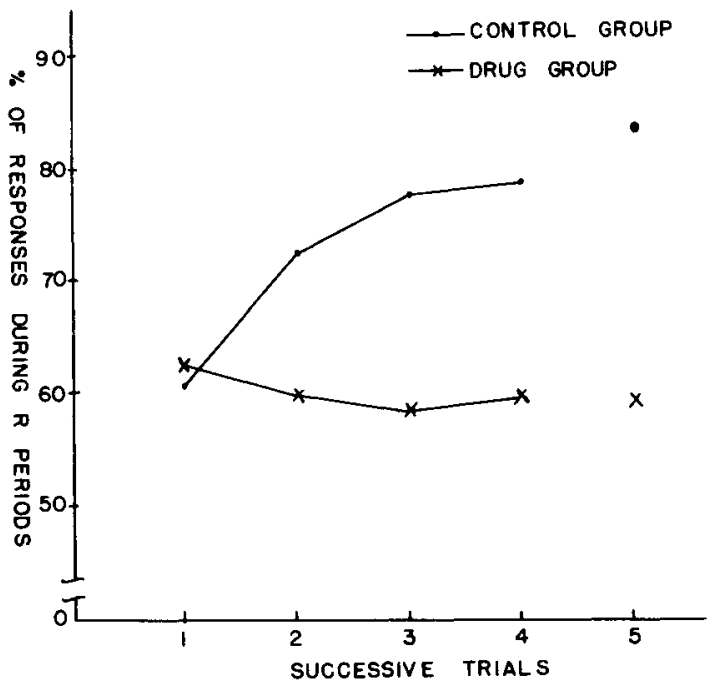

Fig. 2. Mean percent of responses made during $R$ periods by drug and control groups on successive trials.
The significant Drug by Trials interaction effect on overall operant performance (see Fig. 1, Trials 1-4) indicates that the depressant effect of the drug varied over trials, and when compared with the performance of the control Ss, seemed to demonstrate an increased effectiveness. Since drug Ss and control Ss received the same $R$ and NR program, the change in effectiveness cannot be attributed to variations in schedules, but may reflect a cumulative action of the drug. Although both groups demonstrated considerable variability in performance over Trials $1-4$, the recovery data on Trial 5 indicate the depression of operant performance was due to the drug and not to other conditions operating over trials.

The present study also demonstrates the retarding influence of amphetamine on food-motivated discrimination even when a visual cue is present to facilitate the discrimination (see Fig. 2, Trials 1-4). However, a look at the discriminatory data on Trial 5 indicates an entirely different picture than that observed with Trial 5 overall operant rate. Whereas nearly a full recovery occurred in operant performance with distilled water, no such immediate improvement in food-motivated discrimination was observed;i.e., there was no increase in percent of responses during $\mathbf{R}$ periods. These findings suggest that amphetamine specifically retarded the learning of discrimination rather than temporarily repressing discrimination as a mere by-product of its effect on overall operant rate. Consistent with this assumption is the expectation that a gradual improvement in the discrimination of the drug Ss (similar to that of the control Ss with better than $80 \%$ discrimination observed on Trial 5) would occur with additional distilled water trials.

Further investigation is needed to determine the relationship of this retarding action of amphetamine on food-motivated discrimination learning to the more general anorexic properties of the drug.

\section{References}

COLE, S. O. Experimental effects of amphetamine: A review. Psychol Bull., 1967, 68, 81-90.

FERSTER, C. B., APPEL, J. B., \& HISS, R. A. The effect of drugs on a fixed-ratio performance suppressed by a pre-time-out stimulus. $J$. exp. Anal. Behav., 1962, 5, 73-88.

KELLEHER, R. T., FRY, W., DEEGAN, J., \& COOK, L. Effects of meprobamate on operant behavior of rats. J. Pharmacol. exp. Ther. 1961, 133, 271-280.

Notes

1. South Jersey campus, Camden, New Jersey 08102.

2. Purchased from the P. J. Noyes Co., Lancaster, New Hampshire. 3. Supplied by Smith, Kline and French Labs., Philadelphia, Pa. 